\title{
Medical Students and Interventional Radiology: Miles to Go
}

\author{
Rengarajan Rajagopal ${ }^{1}$ Pushpinder Singh Khera ${ }^{2}$ \\ ${ }^{1}$ Department of Cardiovascular Radiology \& Endovascular \\ interventions, All India Institute of Medical Sciences, New Delhi, \\ India \\ 2Department of Diagnostic \& Interventional Radiology, All India \\ Institute of Medical Sciences, Jodhpur, Rajasthan, India
}

J Clin Interv Radiol ISVIR 2018;2:206

We read with interest the editorial titled 'Medical students and interventional radiology.' ${ }^{1}$ The importance of educating undergraduate medical students about interventional radiology (IR) could be stressed in no lesser words.

In this context, we would like present the results of a survey we had conducted among undergraduate medical students 2 years ago at a tertiary healthcare hospital with in-house interventional radiological services. The survey questionnaire consisted of 10 questions with multiple-choice options, which were administered after obtaining an informed consent from 48 students before their first clinical rotation in radiology. The survey presented some glaring and interesting results. Sixty-eight percent of the participants answered 'no' to the question on whether they were aware that radiologists could offer treatment to patients in addition to diagnostic services. About $80 \%$ of the participants had never seen an interventional radiological procedure being performed.

For the question on the most common procedure that was performed by interventional radiologists, $56 \%$ of participants answered as 'image-guided biopsy' followed by 30\% answering as 'guided drain placement.' Seventy-seven percent of participants were not aware of treatment options other than chemotherapy, surgery, and radiation that could be offered to cancer patients. While only $48 \%$ of participants said that radiologists had a role in the emergency room, only $30 \%$ of these participants were aware of roles other than diagnostic services. Eighty percent of participants were not aware of interventional radiological procedures for treatment of hemoptysis. Seventy percent of participants answered that angiographies were performed by cardiologists, while only $22 \%$ of the participants were aware that radiologists too can perform angiography. 'Coronary angiography' was the word that came to the mind of more than $90 \%$ of the participants, while $10 \%$ answered as 'cerebral angiography,' and none of the participants was aware of renal angiography for renal vascular disease. The results of this survey reflect the general lack of awareness about interventional radiological procedures among undergraduate medical students, who in their following years will become experts in different specialities.

\begin{abstract}
Address for correspondence Rengarajan Rajagopal, MD, Room No. 10A, Department of Cardiovascular Radiology \& Endovascular Interventions, All India Institute of Medical Sciences, Ansari Nagar,New Delhi 110029, India (e-mail: heraghava@gmail.com).
\end{abstract}

Interventional radiological procedures have become an integral part of treating patients including cancer patients. Interventional oncology is rapidly gaining popularity as the fourth pillar of cancer treatment. Many interventional radiological procedures such as stent graft placement (e.g., for acute complicated aortic dissection, or aneurysm exclusion, or atherosclerotic occlusive disease), embolization for bleeding (e.g., hemoptysis, or gastrointestinal bleeding, or trauma), and therapeutic drainage of obstructed biliary or urological systems have become the standard of care for patients.

The onus of introducing these procedures to undergraduate medical students lies with the interventional radiologist fraternity. The presence of interventional radiologists in combined clinical rounds and their active participation in multidisciplinary treatment boards would increase the awareness about interventional procedures both among their physician colleagues and medical students. Integrated teaching sessions on specific clinical situations and their nonsurgical and nonpharmacological treatment options including interventional procedures could improve awareness among undergraduate medical students. Finally, we second the statement that IR as a specialty should make efforts to expose medical students to interventional radiological procedures to improve patient care as well as to inspire new young minds to take IR as a specialty.

Ethical Clearance
Obtained from IEC.

Meeting Presentations

None.

Conflicts of Interest

None.

Acknowledgments

None.

\section{Reference}

1 Keshava SN, Palumbo A. Medical students and interventional radiology. J Clin Interv Radiol 2018;2(1):1
License terms

Vascular and Interventional Radiology
June 29, 2018 accepted after revision July 16, 2018 published online November 27, 2018
DOI https://doi.org/

10.1055/s-0038-1675885 ISSN 2457-0214.
()(1) $\odot \circledast$ 